\title{
Morphology-controlled Pd nanocrystals as catalysts in tandem dehydrogenation-hydrogenation reactions
}

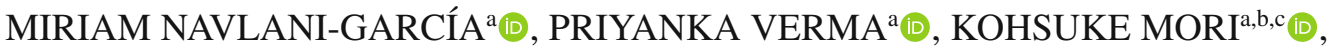 \\ YASUTAKA KUWAHARA ${ }^{\mathrm{a}, \mathrm{b}}$ (D) and HIROMI YAMASHITA ${ }^{\mathrm{a}, \mathrm{b}, *}$ (D) \\ ${ }^{a}$ Division of Materials and Manufacturing Science, Graduate School of Engineering, Osaka University, \\ 2-1 Yamada-oka, Suita, Japan \\ ${ }^{b}$ Unit of Elements Strategy Initiative for Catalysts \& Batteries, Kyoto University, Katsura, Kyoto 615-8520, \\ Japan \\ ${ }^{\mathrm{c}}$ JST, PRESTO, 4-1-8 Honcho, Kawaguchi, Saitama, Japan \\ E-mail: yamashita@mat.eng.osaka-u.ac.jp
}

MS received 11 April 2017; revised 16 July 2017; accepted 25 July 2017; published online 22 September 2017

\begin{abstract}
A facile synthetic protocol was used to prepare morphology controlled Pd nanocrystals with spherical and cubic shapes of different sizes. Carbon-supported catalysts were prepared from the as-synthesised nanocrystals and their catalytic ability in a tandem dehydrogenation/hydrogenation reaction composed by the dehydrogenation of ammonia borane, serving as a hydrogen source, and the subsequent hydrogenation of 4 nitrophenol (4-NP) to 4-aminophenol (4-AP) was assessed. The catalytic performance was strongly dependent on the nanocrystals morphology and the spherical nanoparticles with an average size of $5.5 \mathrm{~nm}$ displayed the best performance among investigated.
\end{abstract}

Keywords. Pd nanocrystals; morphology control; 4-nitrophenol hydrogenation; tandem reactions.

\section{Introduction}

The development of nanomaterials via either bottom-up or top-down approaches has been the central point of decades of research studies, ${ }^{1}$ as they show features that significantly differ from those of the bulk counterparts. Among the studied nanomaterials, metal nanocrystals constitute without a shadow of doubt one of the most investigated and reported groups, due to their repercussion in the field of catalysis. Metal nanocrystals, with a high surface-to-volume ratio, are pivotal in catalysis, as they tend to create more catalytically active sites than bulk materials, as they show low coordinated atoms, usually located in the defects, such as terraces, edges, kinks and vacancies. In general terms, the catalytic activity of metal nanocrystals has demonstrated to be strongly dependent on their morphology, in terms of both nanocrystal size and shape, which has boosted the design of synthetic methodologies that allow the careful control of such morphological properties. More specifically, important breakthroughs have been achieved by using colloidal synthetic strategies that allow the control of the morphology of the nanocrystals in a step previous to their loading on the desired support. ${ }^{2-4}$ Among noble metals, the outstanding catalytic ability showed by $\mathrm{Pd}$ in reactions of different nature, such as hydrogenation/dehydrogenation reactions, ${ }^{5-9}$ hydrogen purification, ${ }^{10,11}$ organic pollutant removal, ${ }^{12}$ and a wide range of organic reactions, ${ }^{13-15}$ has made it deserving of numerous studies dealing with the control of the Pd nanocrystals morphology. ${ }^{16-20}$ Among these reactions, the performances displayed by $\mathrm{Pd}$ in dehydrogenation and hydrogenation reactions should be highlighted. ${ }^{21-23}$ In this study, we assess the catalytic activity of Pd nanocrystals with different morphologies in a tandem dehydrogenation/hydrogenation reaction composed by the dehydrogenation of ammonia borane $\left(\mathrm{NH}_{3} \mathrm{BH}_{3}\right)$, serving as a hydrogen source, and the

\footnotetext{
*For correspondence

Electronic supplementary material: The online version of this article (https://doi.org/10.1007/s12039-017-1370-7) contains supplementary material, which is available to authorized users.
} 
subsequent hydrogenation of 4-nitrophenol (4-NP) to 4-aminophenol (4-AP). The synthesis of 4-AP is of great importance and the global consumption is enormous and increases at a rate of $5 \%$ per year, as it is crucial in the pharmaceutical industry as an intermediate for the synthesis of analgesic and antipyretic drugs, such as paracetamol and acetaminophen ${ }^{24,25}$ and it can be also used in diverse applications such as corrosion inhibitors, photographic developers and hairdyeing agent. ${ }^{26}$ However, the importance of this reaction does not lie exclusively in the production of a valuable chemical, but also in the conversion of a toxic pollutant that affects the human health and environment and that is not easily biodegraded. ${ }^{26,27}$ The morphology dependence of the 4-NP reduction was analysed by using spherical and cubic carbon-supported Pd nanocrystals of different sizes (spheres: 2.7, 3.9 and $5.5 \mathrm{~nm}$; cubes: 7.6, 10.3 and $12.6 \mathrm{~nm}$ ). To this end, the shaped nanocrystals were initially synthetised in colloidal form and subsequently deposited on the support by following a standard impregnation method.

\section{Experimental}

\subsection{Preparation of $P d$ spheres}

Colloidal spherical Pd nanoparticles with tuned size were synthesised by using the polyol method by a methodology reported elsewhere. ${ }^{7,8,28}$ To this end, palladium (II) acetate was used as Pd precursor, polyvinylpyrrolidone (PVP, K-30) as a stabilising agent and ethylene glycol as a solvent and reductant. To conduct the nanoparticles synthesis, two solutions were prepared. Solution 1, containing PVP and ethylene glycol $(30 \mathrm{~mL})$, was stirred at $80^{\circ} \mathrm{C}$ for $2 \mathrm{~h}$, while solution 2 , containing Pd precursor dissolved in dioxane $(12.5 \mathrm{~mL})$, was stirred at room temperature. Solution 1 was cooled down to $0^{\circ} \mathrm{C}$ and $0.75 \mathrm{~mL}$ of $1 \mathrm{M} \mathrm{NaOH}$ solution was added under stirring to adjust the $\mathrm{pH}$ of the resulting mixture. After that, solution 2 was poured into solution 1 under vigorous stirring and the mixture was heated up to $100^{\circ} \mathrm{C}$. The solution changed its colour from light orange to dark brown due to the reduction of $\mathrm{Pd}(\mathrm{II})$ to $\mathrm{Pd}(0)$. The heating was maintained for $2 \mathrm{~h}$, and then the colloidal suspension was cooled down to room temperature. In order to remove the excess of PVP from the nanoparticles surface, an excess of acetone was added to the colloid and the solution was shaken vigorously, causing the extraction of PVP to the acetone phase and flocculation of the Pd nanoparticles. The purified nanoparticles were separated from the organic phase and redispersed by stirring in a known amount of methanol. The nanoparticles size was controlled by selecting various PVP/Pd ratios and Pd precursor concentration in the initial solution (Table 1).

\subsection{Preparation of $P d$ cubes}

The preparation of Pd cubes was achieved by means of colloidal synthesis in aqueous phase by using $\mathrm{Na}_{2} \mathrm{PdCl}_{4}$ as $\mathrm{Pd}$ precursor, $\mathrm{L}$-ascorbic acid (AA) as a reductant agent, $\mathrm{PVP}$ as a stabiliser, and halide ions $\left(\mathrm{Br}^{-}\right.$or $\left.\mathrm{Br}^{-}+\mathrm{Cl}^{-}\right)$as a capping agent to stabilise a specific facet on the Pd nanocrystals surface. ${ }^{16,29} \mathrm{AA}, \mathrm{PVP}$ and halide ions were dissolved in $16 \mathrm{~mL}$ of water and the solution was heated at $80^{\circ} \mathrm{C}$ for $10 \mathrm{~min}$ while stirring. Afterwards, the Pd precursor was added to the solution and the temperature was kept for $3 \mathrm{~h}$. Then, an excess of acetone was added to the slurry and the nanocrystals were collected by centrifugation, washed in distilled water and redispersed in the same solvent. The amount of reagents used for the synthesis of three colloids with different average size is listed in Table 1.

\subsection{Preparation of carbon supported catalysts}

Six carbon-supported catalysts were prepared from the Pd colloids by using the impregnation method. Commercial SHIRASAGI M (Osaka Gas Chemicals Co. Ltd.) carbon support was impregnated with the adequate amount of Pd colloid so as to result in a $0.5 \mathrm{wt} \%$ of Pd. The catalysts are hereinafter denoted as C-Spheres (S), C-Spheres (M), C-Spheres (L), CCubes (S), C-Cubes (M) and C-Cubes (L).

\subsection{Characterisation}

Porous texture of the catalysts was characterised by means of adsorption of $\mathrm{N}_{2}$ at $-196^{\circ} \mathrm{C}$ by using a BELSORP-max

Table 1. Experimental conditions used in the synthesis of sized-controlled Pd nanocrystals with spherical and cubic shapes.

\begin{tabular}{lccccc}
\hline Pd colloid & PVP/Pd Molar ratio [a] & Pd precursor (mmol) & AA (mmol) & KBr (mmol) & KCl (mmol) \\
\hline Spheres (S) & 10 & 0.062 & - & - & - \\
Spheres (M) & 10 & 0.250 & - & - & - \\
Spheres (L) & 1 & 0.250 & - & - & - \\
Cubes (S) & 5 & 0.380 & 0.670 & 1.0 & 4.9 \\
Cubes (M) & 10 & 0.390 & 0.680 & 5.0 & - \\
Cubes (L) & 5 & 0.390 & 0.680 & 5.0 & - \\
\hline
\end{tabular}

Note: $\mathrm{S}, \mathrm{M}$ and $\mathrm{L}$ are referred to small, medium and large average nanocrystals size.

[a] Considering the molecular weight $\left(\mathrm{M}_{W}\right)$ of one monomer unit of $\mathrm{PVP}\left(\mathrm{C}_{6} \mathrm{H}_{9} \mathrm{NO}\right)$. 
system (MicrotracBEL). Before gas adsorption, the catalysts were degassed under vacuum at $250^{\circ} \mathrm{C}$ for $4 \mathrm{~h}$ to remove any adsorbed impurities. Apparent surface area values $\left(\mathrm{S}_{\mathrm{BET}}\right)$ were calculated from $\mathrm{N}_{2}$ adsorption isotherms using the BET equation. Total micropore volume $\left(\mathrm{V}_{\mathrm{DR}}\left(\mathrm{N}_{2}\right)\right)$ was calculated applying the Dubinin-Radushkevich (DR) equation to the $\mathrm{N}_{2}$ adsorption data. Transmission Electron Microscopy (TEM) was used to determine the average nanocrystals size of both colloids and supported nanocrystals. Pd 3d X-ray photoelectron spectroscopy (XPS) data were collected using an ESCA 3400 Electron Spectrometer and the relative proportion of $\operatorname{Pd}(0)$ and $\operatorname{Pd}(\mathrm{II})$ was determined by the integrated intensities of the spectra.

\subsection{Reduction of 4-nitrophenol to 4-aminophenol by ammonia borane}

An aqueous solution of 4-NP $(0.017 \mathrm{M})$ and $\mathrm{NH}_{3} \mathrm{BH}_{3}(0.13$ $\mathrm{M})$ were freshly prepared. The carbon-supported Pd catalyst powder $(0.4 \mathrm{mg})$ and $4-\mathrm{NP}$ solution $(40 \mu \mathrm{L})$ were mixed in a test tube together with distilled water $(3 \mathrm{~mL}) .20 \mu \mathrm{L}$ of the $\mathrm{NH}_{3} \mathrm{BH}_{3}$ solution was added to start the reaction. The initial 4-NP UV-Vis spectrum shows an absorption peak at 317 $\mathrm{nm}$ and upon $\mathrm{NH}_{3} \mathrm{BH}_{3}$ addition, a peak a $400 \mathrm{~nm}$ appears caused by the formation of 4-NP anions by $\mathrm{H}^{+}$dissociation from 4-NP in basic $\mathrm{NH}_{3} \mathrm{BH}_{3}$ solution. The evolution of the 4-NP hydrogenation was monitored by checking the decreasing intensity of this peak at $400 \mathrm{~nm}$ together with the increasing intensity of a new peak at $296 \mathrm{~nm}$, which is due to the formation of 4-AP. ${ }^{30,31}$ It should be pointed out that due to the high activity showed by most of the samples, the amount of $\mathrm{NH}_{3} \mathrm{BH}_{3}$ added to start the reaction was adjusted to $20 \mu \mathrm{L}$ of the prepared solution $(0.13 \mathrm{M})$, which is low as compared with other works where the same reaction was studied. In order to determine which is the limiting reaction step in the tandem dehydrogenation/hydrogenation reaction, the $\mathrm{NH}_{3} \mathrm{BH}_{3}$ decomposition reaction was also monitored for some of the catalysts. In this case, $10 \mathrm{mg}$ of catalyst and $5 \mathrm{~mL}$ of water were mixed in a Pyrex reaction vessel $(30 \mathrm{~mL})$ and the mixture was treated in an ultrasound bath for $15 \mathrm{~min}$ to fully disperse the catalyst. Once it was dispersed, the mixture was bubbled with argon gas for $15 \mathrm{~min}$. To start the reaction, $50 \mu \mathrm{L}$ of $\mathrm{NH}_{3} \mathrm{BH}_{3}(0.5 \mathrm{M})$ was added into the reactor and the hydrogen production was monitored by means of gas chromatography using a Shimadzu GC14B equipped with MS5 A column.

\section{Results and Discussion}

\subsection{Characterisation of the catalysts}

$\mathrm{N}_{2}$ adsorption-desorption isotherms at $-196^{\circ} \mathrm{C}$ for all the catalysts as well as the carbon support are displayed in Figure S1. As it can be seen from the shape of the isotherms, all the samples showed a Type I isotherm, indicating the microporous nature of the carbon material used. ${ }^{32}$ Table S1 shows the results of the porous texture characterisation. In all cases, the specific surface area of the raw support is slightly decreased after the loading of Pd nanocrystals, being this effect more pronounced for the smallest nanocrystals in both series of catalysts, spheres and cubes, which might be due to a more important blocking of the carbon porosity by those nanocrystals with small average size. TEM micrographs for Pd spheres and cubic nanocrystals in both colloidal form and carbon-supported catalysts, together with their corresponding size distribution diagrams, are shown in Figures 1 and 2, and the average nanocrystals size determined by counting $\sim 100$ nanocrystals is listed in Table 2.

As it can be seen from the result in Table 2, in the case of the spherical nanoparticles, the average nanoparticle size was 1.9, 2.1 and $3.2 \mathrm{~nm}$ for $\operatorname{Spheres}(\mathrm{S})$, (M) and (L), respectively, while for the colloidal cubes it was 6.9, 9.7 and $10.3 \mathrm{~nm}$ for Cubes(S), (M) and (L), respectively. It should be noticed that while the control of the spherical nanoparticles size is relatively easy by using colloidal synthesis, the preparation of small $\mathrm{Pd}$ nanocubes is very difficult. Most of the studies found in the literature reported the synthesis of cubes as large as $10 \mathrm{~nm}$ or larger. ${ }^{33-36}$ Nevertheless, both series of samples showed narrow nanocrystal size distributions, especially in the case of Pd spheres. Furthermore, both the series of colloids showed well-defined shapes and pure spheres and cubes were achieved, respectively, and no other morphologies were obtained in these cases. This high purity was achieved by the adjustment of the amount of the agent used in the synthesis and may be partially lost by slightly modifying their relative proportion in the synthesis. For instance, a colloid containing spheres, cubes and rods were synthesised by adding a lower proportion of $\mathrm{KBr}$ in the synthesis of nanocubes, while keeping all the experimental conditions used in the synthesis of Cubes(S) (Figure S2). The nanocrystals do not agglomerate after being loaded onto the support, but they experienced an apparent increase in their size, which might be due to their interaction with the carbon support. It should be noted that this increase is much more pronounced in the case of spherical nanoparticles, which might be primarily attributed to their higher surface roughness as compared to the nanocubes, that in turn is related to the facet exposed by both types of morphologies (spherical nanoparticles have been reported to be considered as a combination of low- and high-index facets (terraces: $\{111\},\{100\}$; steps: $\{331\},\{443\}$, etc.; kinks: $\{10,8,7\},\{13,11,9\}$, etc., and corner sites and adatoms, etc.; while cubic nanocrystals are enclosed exclusively with six low-index $\operatorname{Pd}\{100\}$ facets). ${ }^{37,38}$ 


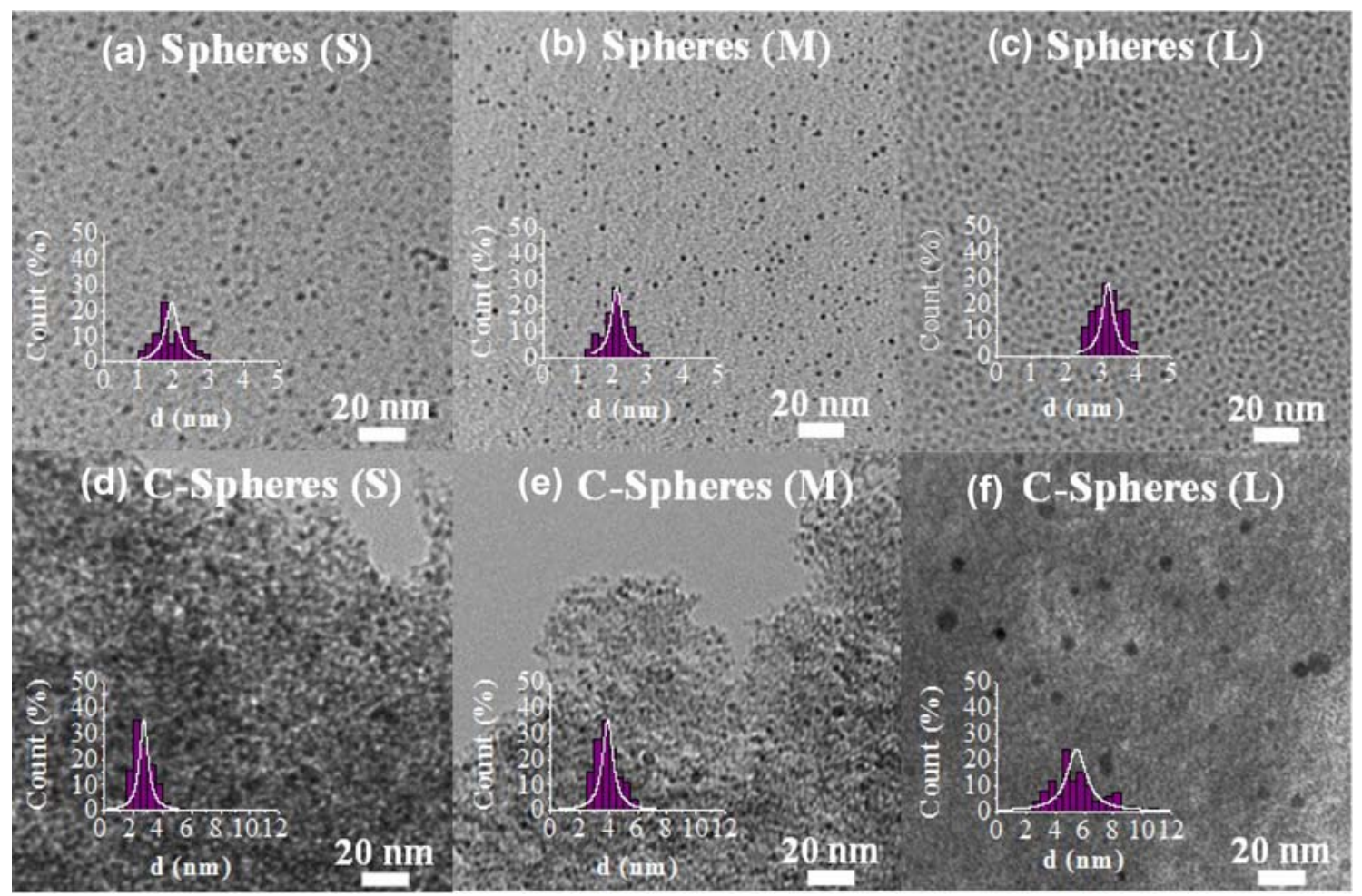

Figure 1. TEM images and the corresponding histograms for the Pd colloid with spherical shape ((a) Spheres (S), (b) Spheres (M) and (c) Spheres (L)) and the carbon-supported catalysts counterparts ((d) C-Spheres (S), (e) C-Spheres (M) and (f) C-Spheres (L)).

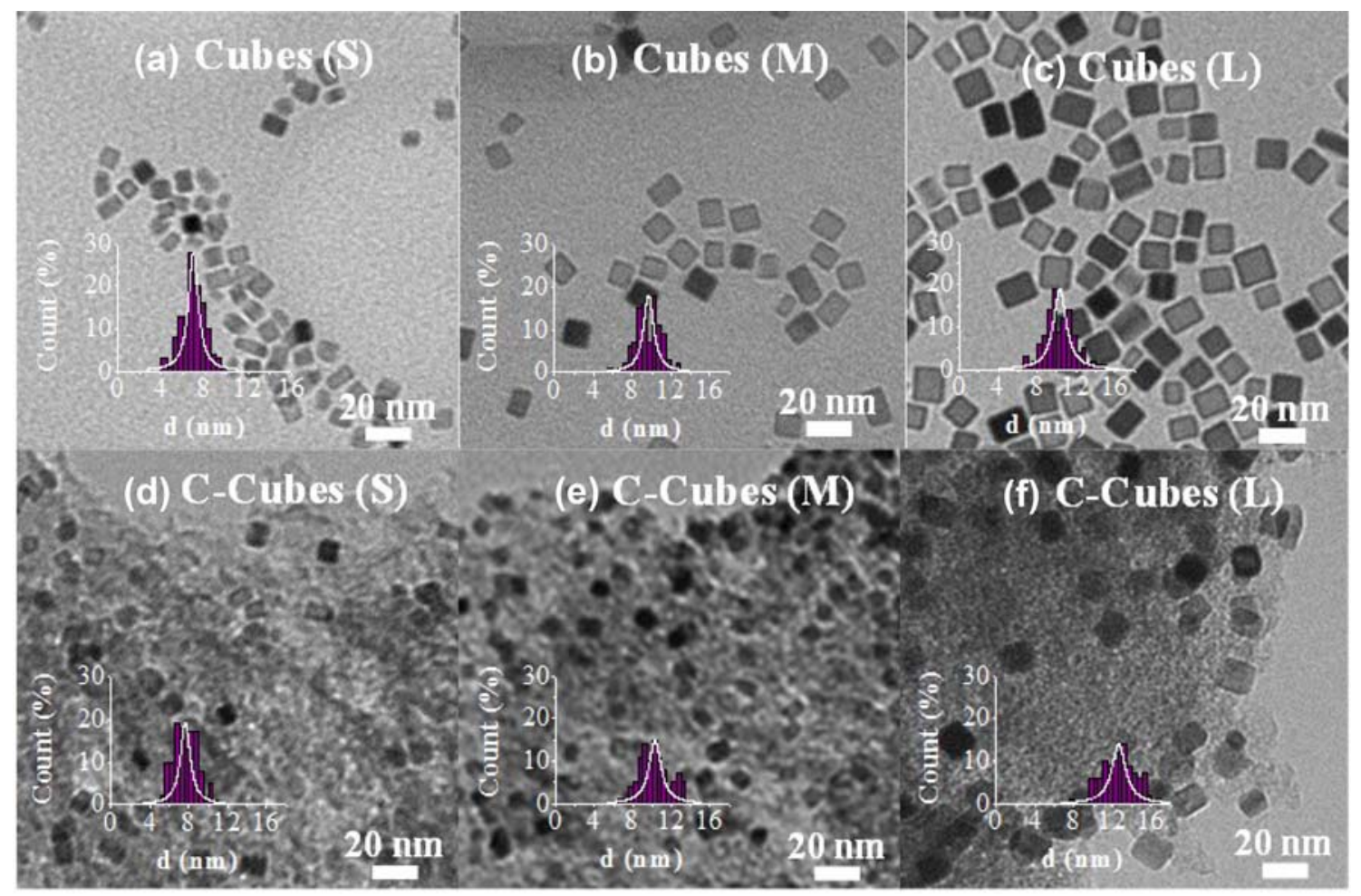

Figure 2. TEM images and the corresponding histograms for the Pd colloid with cubic shape ((a) Cubes (S), (b) Cubes (M) and (c) Cube (L)) and the carbon-supported catalysts counterparts ((d) C- Cubes (S), (e) C-Cubes (M) and (f) C-Cubes (L)). 
Table 2. Average Pd nanocrystals size in the colloids and pertinent carbon-supported catalysts.

\begin{tabular}{lccc}
\hline Colloid & $\mathrm{d}(\mathrm{nm})$ & Catalyst & $\mathrm{d}(\mathrm{nm})$ \\
\hline Spheres (S) & $1.9 \pm 0.4$ & C-Spheres (S) & $2.7 \pm 0.7$ \\
Spheres (M) & $2.1 \pm 0.4$ & C-Spheres (M) & $3.9 \pm 0.9$ \\
Spheres (L) & $3.2 \pm 0.4$ & C-Spheres (L) & $5.5 \pm 1.6$ \\
Cubes (S) & $6.9 \pm 1.1$ & C-Cubes (S) & $7.6 \pm 1.2$ \\
Cubes (M) & $9.7 \pm 1.3$ & C-Cubes (M) & $10.3 \pm 1.5$ \\
Cubes (L) & $10.3 \pm 1.6$ & C-Cubes (L) & $12.6 \pm 1.7$ \\
\hline
\end{tabular}

Table 3. XPS analysis results.

\begin{tabular}{|c|c|c|}
\hline Sample & $\operatorname{Pd}(0) \quad$ atomic \%) & $\mathrm{Pd}(\mathrm{II})$ (atomic \%) \\
\hline C-Spheres (S) & 84.1 & 15.9 \\
\hline C-Spheres (M) & 83.3 & 16.7 \\
\hline C-Spheres (L) & 81.5 & 18.5 \\
\hline C-Cubes (S) & 77.4 & 22.6 \\
\hline C-Cubes (M) & 84.1 & 15.9 \\
\hline C-Cubes (L) & 80.7 & 19.3 \\
\hline
\end{tabular}

This higher roughness might favour the interaction with the carbon support and the subsequent flattening of the spherical nanoparticles that is ultimately observed by an apparent increase in their average nanoparticles size. Concerning the supported nanocrystals shape, it was slightly affected by the immobilisation procedure onto the support, which was particularly observed in the case of sample C-Cubes (S), where some rounded corner cubes were detected but complete transformation from cubic to spherical nanoparticles was not seen. This partial modification of cubic nanocrystals after the immobilisation steps has been previously observed by other authors, who also perceived that smaller cubes were less stable against shape modification than the larger counterparts. ${ }^{39}$

Concerning Pd electronic features, XPS analysis was conducted to determine the relative proportion of metallic and oxidised species. The spectra recorded for all samples displayed two electronic transitions attributed to the bands of $\mathrm{Pd} 3 \mathrm{~d}_{5 / 2}$ and $3 \mathrm{~d}_{3 / 2}$ (at lower and higher binding energies, respectively). Each of these bands is deconvoluted into two bands corresponding to different $\mathrm{Pd}$ species, $\operatorname{Pd}(0)$ represented by the peak at lower binding energy and $\mathrm{Pd}(\mathrm{II})$ and higher binding energy. ${ }^{8}$ The quantitative analyses were done from the integrated intensities of the spectra (See Figure S3 and Table 3 for details).

As it can be seen, both $\operatorname{Pd}(0)$ and $\mathrm{Pd}(\mathrm{II})$ were present in all the catalysts. The presence of Pd(II) in the surface of Pd nanocrystals might be partially due to the existence of PVP linked to their surface. As it was previously reported in previous studies reported by our research group,${ }^{40}$ the interaction between the nanoparticles surface and PVP leads to the withdrawing electron density from the metal surface by the $\mathrm{CO}$ functional group present in the polymer, which causes the presence of $\mathrm{Pd} \delta^{+}$on the nanoparticles surface and that is observed as Pd (II) in XPS spectra. Nevertheless, such $\mathrm{Pd}(\mathrm{II})$ surface species might be reduced during the initial stage of the catalytic test and therefore, the effect of the Pd electronic features will be ruled out in this study.

\subsection{Catalytic tests}

In this work, we followed the evolution of the 4-NP reduction to 4-AP through a tandem dehydrogenation/hydrogenation reaction by using $\mathrm{NH}_{3} \mathrm{BH}_{3}$ as a hydrogen source and carbon-supported $\mathrm{Pd}$ catalysts as dual role catalysts to boost the dehydrogenation of $\mathrm{NH}_{3} \mathrm{BH}_{3}$ and the subsequent hydrogenation of 4-NP with the hydrogen generated from the first reaction. In a first step, we checked the ability of the studied catalysts towards the hydrogenation of 4-NP by using $\mathrm{NaBH}_{4}$ and $\mathrm{NH}_{3} \mathrm{BH}_{3}$ as a hydrogen source and those preliminary tests revealed that, under the experimental conditions used in this case, $\mathrm{NH}_{3} \mathrm{BH}_{3}$ was more suitable to conduct these experiments because its dehydrogenation proceeded more easily. The comparative catalytic performance of C-Spheres and C-Cubes is displayed in Figure 3. According to the UV-Vis spectra recorded at different reaction times, reduction of 4-NP was catalysed by all the analysed samples, as indicated by the decrease in the absorption of 4-NP peak at 400 $\mathrm{nm}$ together with the concomitant increase of the peak corresponding to the presence of 4-AP at $296 \mathrm{~nm}$. Nevertheless, important differences were appreciated for both different shapes and sizes. In the case of the CSpheres, complete reduction of 4-NP was achieved by using medium or large nanoparticles, while the reduction was not completed while testing C-Sphere (S). The time needed for the total reduction of 4-NP was 16.5 and $10.5 \mathrm{~min}$, for C-Spheres (M) and (L), respectively, which revealed the better performance achieved by the larger Pd nanoparticles with a spherical shape. A dissimilar trend was attained with the C-Cubes set of samples. In this case, completely 4-NP reduction took place only with $\mathrm{C}$-Cubes $(\mathrm{M})$, and the reaction was not completed for smaller or larger cubes, even for longer reaction times (25.0 and $28.5 \mathrm{~min}$ for $\mathrm{C}$-Cubes (S) and (L), respectively, compared to $18.0 \mathrm{~min}$ needed by CCubes (M) to catalyse the total 4-NP hydrogenation). It should be noticed that for both the sets of samples, the poorest performance in terms of reaction conversion 

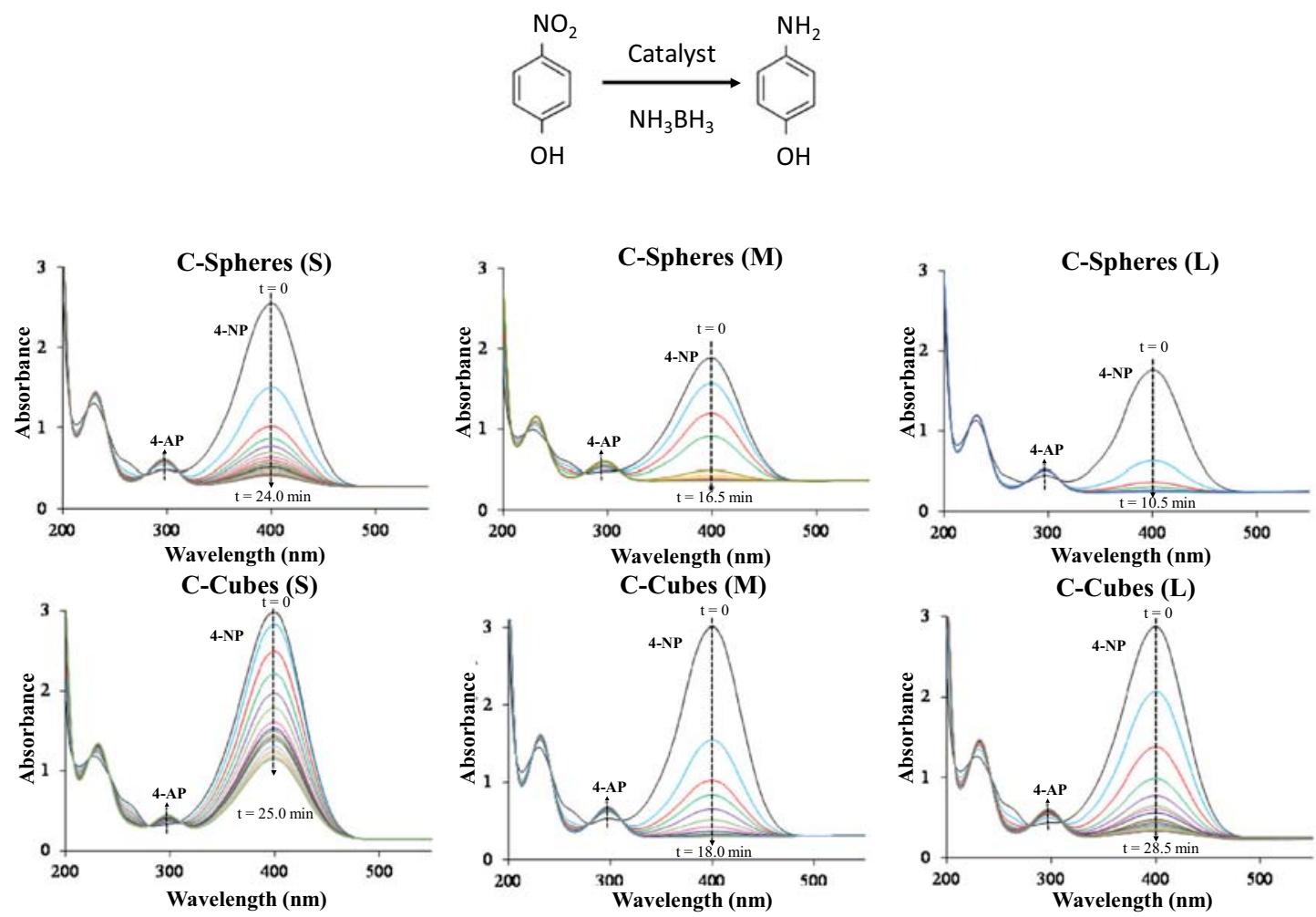

Figure 3. UV-Vis absorption spectra of the reduction of 4-NP catalysed by carbon-supported Pd spheres and cubes with different nanocrystal size. Initial 4-NP concentration of $0.2 \mathrm{mM}$ and optical path length of 1 $\mathrm{cm}$.

(a) C-Spheres

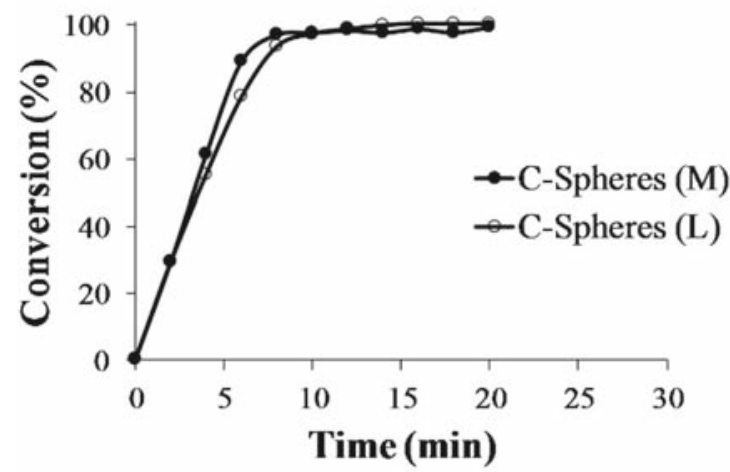

(b) C-Cubes

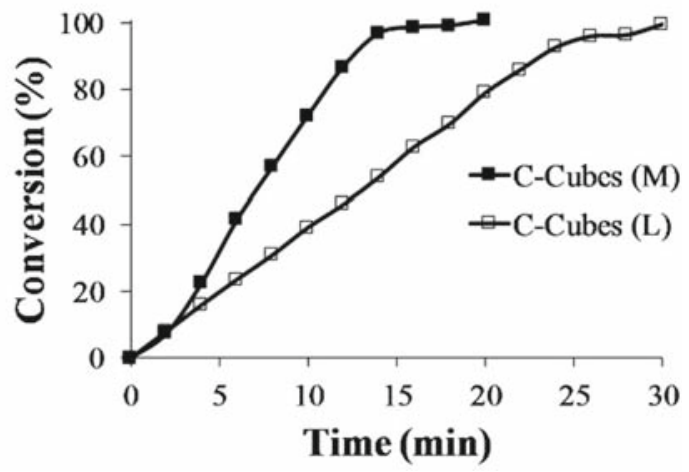

Figure 4. Time dependence of $\mathrm{NH}_{3} \mathrm{BH}_{3}$ conversion to produce $\mathrm{H}_{2}$ for (a) C-Spheres and (b) C-Cubes.

(considering $100 \%$ of conversion for total extinction of the absorption peak of 4-NP at $400 \mathrm{~nm}$ ) was observed in the case of C-Spheres (S) and C-Cubes (S), which is contrary to the general better performance of smaller nanocrystals with a larger proportion of coordinately unsaturated metal atoms observed in some catalytic reactions. ${ }^{41-43}$

As the hydrogenation of 4-NP proceeds by using the hydrogen generated from the $\mathrm{NH}_{3} \mathrm{BH}_{3}$ dehydrogenation reaction, the final performance is determined by both reactions. Then, to get further insight into the morphology sensitivity of the studied catalytic system, $\mathrm{NH}_{3} \mathrm{BH}_{3}$ dehydrogenation reaction was monitored for the two best-performing catalysts of both sets of samples (i.e., C-Spheres (M) and (L); C-Cubes (M) and (L)). This dehydrogenation proceeds according to the following reaction: $\mathrm{NH}_{3}-\mathrm{BH}_{3}+2 \mathrm{H}_{2} \mathrm{O} \rightarrow \mathrm{NH}_{4} \mathrm{BO}_{2}+3 \mathrm{H}_{2}$; so $100 \%$ of conversion means the generation of $3 \mathrm{~mol}$ of 
$\mathrm{H}_{2}$ per mol of $\mathrm{NH}_{3} \mathrm{BH}_{3}$. The time dependence $\mathrm{NH}_{3} \mathrm{BH}_{3}$ conversion profiles are plotted in Figure 4. Figure 4 (a) revealed that, under the experimental conditions used in these tests, the $\mathrm{NH}_{3} \mathrm{BH}_{3}$ dehydrogenation did not show a marked size-dependency for the $\mathrm{C}$-Spheres samples in the nanoparticle size range under study. However, an important size-dependence was seen for C-Cubes. In this case, the reaction was more efficiently boosted by sample C-Cubes (M) $(\sim 100 \%$ conversion achieved after 14 and 26 min of reaction for C-Cubes (M) and C-Cubes (L), respectively). Furthermore, from a comparative point of view, $\mathrm{C}$-Spheres assisted more efficiently the $\mathrm{NH}_{3} \mathrm{BH}_{3}$ dehydrogenation than C-Cubes, as only 10 min was needed to achieve $\sim 100 \%$ conversion for both $\mathrm{C}$-Spheres $(\mathrm{M})$ and $(\mathrm{L})$. The better performance of C-Spheres is reflected in the TOF values: 43 and $39 \mathrm{~min}^{-1}$ for $\mathrm{C}$-Spheres $(\mathrm{M})$ and $(\mathrm{L})$, respectively, and 21 and $12 \mathrm{~min}^{-1}$ for C-Cubes (M) and (L), respectively (calculated after $6 \mathrm{~min}$ of reaction in all cases). Then, attending to this observation and, from a general overview, the better behaviour displayed by $\mathrm{C}$-Spheres as compared to C-Cubes in the hydrogenation of 4-NP can be initially ascribed to their superior performance in boosting the $\mathrm{NH}_{3} \mathrm{BH}_{3}$ dehydrogenation, which in turn could be attributed to their smaller average nanocrystal size which affords a more important contribution of surface atoms with respect to the total number of atoms in the nanocrystals (surface atoms account for 35.5, 30.0 and $24.0 \%$ of the total atoms in C-Spheres (S), (M) and (L), respectively, and only 14.5, 10.9 and $9.0 \%$ of the total atoms in C-Cubes (S), (M) and (L), respectively; See Supporting Information for calculation details). Nevertheless, due to the different facets exposed by Pd spheres and cubes, the structurereactivity dependency cannot be ruled out and a more thorough investigation should be conducted to ascertain the main factor in controlling the catalytic activity of the present system.

Moreover, some additional considerations should be kept in mind to elucidate the effect of the nanocrystal size within both sets of catalysts. In this line, it seems that the better catalytic activity displayed by C-Spheres (L) as compared to C-Spheres (S) and (M) cannot be directly and exclusively linked to their ability to catalyse the $\mathrm{NH}_{3} \mathrm{BH}_{3}$ dehydrogenation. From a first approximation, it could be thought that this size-sensitivity could be related to adsorption-desorption phenomena taking place on the nanoparticles surface. Then, the enhancement observed with the increase of the nanoparticle size might be associated to the less important steric hindrance on larger nanoparticles during the adsorption of reagents on the nanoparticles surface to afford the reduction of the large molecules of 4-NP. In the case of Pd cubes, the best-performing catalyst among investigated was $\mathrm{C}$-Cubes (M). The volcano type relationship between the average nanocrystal size and the catalytic ability of these samples might be related to an intermediate surface state between smaller cubic nanocrystals (with more tendency to react) and larger nanocrystals (with less important steric hindrance effect). It should be noted that in this case, the better activity of C-Cubes (M) as compared to larger nanocrystals is in good agreement with their ability to catalyse the $\mathrm{NH}_{3} \mathrm{BH}_{3}$ dehydrogenation reaction, confirming that the final catalytic performance in the tandem reactions is related to the catalyst hydrogen production ability.

\section{Conclusions}

Two sets of carbon-supported catalysts were prepared by means of colloidal synthetic protocols, which allowed the preparation of size and shape controlled Pd spheres and cubes and the subsequent impregnation of the support with the as-synthesised nanocrystals. The resulting catalysts were tested in a tandem dehydrogenation/hydrogenation reaction, in which $\mathrm{NH}_{3} \mathrm{BH}_{3}$ was used as hydrogen source for the subsequent hydrogenation of 4-nitrophenol to 4-aminophenol. In the case of C-Spheres, the catalytic activity enhances as the nanoparticle size increases, which might be related to the favoured surface adsorption/desorption of reagents on the nanoparticles surface due to the less important steric crowding on larger nanoparticles. A different tendency was displayed by C-Cubes, where a volcano type relationship between the nanocrystal size and the catalytic activity was shown.

\section{Supplementary Information (SI)}

Results of the nitrogen adsorption-desorption experiments as well as TEM and XPS characterisation can be found in the SI. Statistics of surface atoms on spheric and cubic Pd nanocrystals are also included. Supplementary Information is available at www.ias.ac.in/chemsci.

\section{Acknowledgements}

The present work was partially supported by Grants-in-Aid for Scientific Research (Nos. 26220911, 25289289, 26630409 and 26620194) from the Japan Society for the Promotion of Science (JSPS) and MEXT. We acknowledge Dr. Eiji Taguchi and Prof. H. Yasuda at the Research Center for Ultra-High Voltage Electron Microscopy, Osaka University, for their assistance with the TEM measurements. KM, YK and HY thank MEXT program "Elements Strategy Initiative to Form Core Research Center". 


\section{References}

1. You H, Yang S, Ding B, and Yang H 2013 Synthesis of colloidal metal and metal alloy nanoparticles for electrochemical energy applications Chem. Soc. Rev. 422880

2. Teranishi T and Miyake M 1998 Size Control of Palladium Nanoparticles and Their Crystal Structures Chem. Mater. 10594

3. Somorjai G A and Park J Y 2008 Colloid science of metal nanoparticle catalysts in 2D and 3D structures. Challenges of nucleation, growth, composition, particle shape, size control and their influence on activity and selectivity Top. Catal. 49126

4. Jia C J and Schüth F 2011 Colloidal metal nanoparticles as a component of designed catalyst Phys. Chem. Chem. Phys. 132457

5. Izadi N, Rashidi A M, Izadi A, Emami S, Samimi V and Varmazyar H 2017 Selective hydrogenation of 4-carboxybenzaldehyde over palladium catalysts supported with different structural organization Int. J. Hydrogen Energy 422970

6. Manna J, Akbayrak S and Özkar S 2017 Palladium(0) nanoparticles supported on polydopamine coated Fe3O4 as magnetically isolable, highly active and reusable catalysts for hydrolytic dehydrogenation of ammonia borane Appl. Catal. B Environ. 208102035

7. Navlani-García M, Martis M, Lozano-Castelló D, Cazorla-Amorós D, Mori K and Yamashita H 2015 Investigation of Pd nanoparticles supported on zeolites for hydrogen production from formic acid dehydrogenation Catal. Sci. Technol. 5364

8. Navlani-García M, Mori K, Nozaki A, Kuwahara Y and Yamashita H 2016 Investigation of Size Sensitivity in the Hydrogen Production from Formic Acid over Carbon-Supported Pd Nanoparticles ChemistrySelect $\mathbf{1}$ 1879

9. García-Aguilar J, Navlani-García M, Berenguer-Murcia A, Mori K, Kuwahara Y, Yamashita H and CazorlaAmoros D 2016 Evolution of the PVP-Pd surface interaction in nanoparticles through the case study of formic acid decomposition Langmuir 3212110

10. Miguel-García I, Navlani-García M, García-Aguilar J, Berenguer-Murcia A, Lozano-Castelló D and CazorlaAmorós D 2015 Capillary microreactors based on hierarchical $\mathrm{SiO}_{2}$ monoliths incorporating noble metal nanoparticles for the Preferential Oxidation of CO Chem. Eng. J. 27574

11. Navlani-García M, Miguel-García I, BerenguerMurcia A, Lozano-Castelló D, Cazorla-Amorós D and Yamashita H 2016 Pd/Zeolite-based catalysts for the Preferential CO Oxidation reaction: ion-exchange, $\mathrm{Si} / \mathrm{Al}$ and structure effect Catal. Sci. Technol. 62623

12. Arciniega Cano O, Rodríguez González C A, Hernández Paz J F, Amezaga Madrid P, García Casillas P E, Martínez Hernández A L and Martínez Pérez C A 2017 Catalytic activity of palladium nanocubes/multiwalled carbon nanotubes structures for methyl orange dye removal Catal. Today 282168

13. Chen Y H, Hung H H and Huang M H 2009 Seedmediated synthesis of palladium nanorods and branched nanocrystals and their use as recyclable suzuki coupling reaction catalysts J. Am. Chem. Soc. 1319114
14. Bharti R, Bal Reddy C, Kumar D and Das P 2017 Supported palladium nanoparticle-catalysed SuzukiMiyaura cross-coupling approach for synthesis of aminoarylbenzosuberene analogues from natural precursor Appl. Organomet. Chem. DOI:10.1002/aoc.3749

15. Fu W, Zhang Z, Zhuang P, Shen J and Ye M 2017 Onepot hydrothermal synthesis of magnetically recoverable palladium/reduced graphene oxide nanocomposites and its catalytic applications in cross-coupling reactions $J$. Colloid Interface Sci. 49783

16. Lim B, Jiang M, Tao J, Camargo P C H, Zhu Y and Xia Y 2009 Shape-controlled synthesis of Pd nanocrystals in aqueous solutions Adv. Funct. Mater. 19189

17. Xiao C, Ding H, Shen C, Yang T, Hui C and Gao H J 2009 Shape-controlled synthesis of palladium nanorods and their magnetic properties J. Phys. Chem. C 11313466

18. Hu Y, Yang X, Cao S, Zhou J, Wu Y, Han Y, Yan Z and Zheng M 2017 Effect of the dispersants on Pd species and catalytic activity of supported palladium catalyst Appl. Surf. Sci. 400148

19. Matsumura S, Sato R, Nakaoka S, Yokotani W, Murakami Y, Kataoka Y and Ura Y 2017 PalladiumCatalyzed Aerobic Synthesis of Terminal Acetals from Vinylarenes Assisted by $\pi$-Acceptor Ligands ChemCatChem 9751

20. Skhiri A, Salem R B, Soulé J F and Doucet H 2017 Unprecedented Access to $\beta$-Arylated Selenophenes through Palladium-Catalysed Direct Arylation Chem. A Eur. J. 232788

21. Zhang W, Wang F, Li X, Liu Y, Liu Y and Ma J 2017 Fabrication of hollow carbon nanospheres introduced with $\mathrm{Fe}$ and $\mathrm{N}$ species immobilized palladium nanoparticles as catalysts for the semihydrogenation of phenylacetylene under mild reaction conditions Appl. Surf. Sci. 404 398

22. Liu G, Liu S, Liu S, Yu S, Li L, Liu F, Xie C and Song X 2017 Hydrogenation of 2-Ethylhexenal Using Supported-Metal Catalysts for Production of 2Ethylhexanol Catal. Lett 147987

23. Li N, Liu M, Yang B, Shu W, Shen Q, Liu M and Zhou J 2017 Enhanced Photocatalytic Performance toward CO2 Hydrogenation over Nanosized $\mathrm{TiO}_{2}$-Loaded Pd under UV Irradiation J. Phys. Chem. C 1212923

24. Chen L, Zhang D and Ge G 2015 A green approach for efficient p-nitrophenol hydrogenation catalyzed by a Pdbased nanocatalyst Catal. Commun. 6695

25. Komatsu T and Hirose T 2004 Gas phase synthesis of para-aminophenol from nitrobenzene on $\mathrm{Pt} /$ zeolite catalysts Appl. Catal. A Gen. 27695

26. Zhao S, Li Q, Li F and Liang Z 2017 Synthesis of spinel $\mathrm{CuCo} 2 \mathrm{O} 4$ nanoparticles and its application in $\mathrm{p}$ nitrophenol reduction J. Sol-Gel Sci. Technol. 81544

27. Park H, Reddy D A, Kim Y, Lee S, Ma R, Lim M and Kim T K 2017 Hydrogenation of 4-nitrophenol to 4aminophenol at room temperature: Boosting palladium nanocrystals efficiency by coupling with copper via liquid phase pulsed laser ablation Appl. Surf. Sci. 401314

28. Navlani-García M, Miguel-García I, Berenguer-Murcia A, Lozano-Castelló D Cazorla-Amorós D and Yamashita H $2016 \mathrm{Pd} /$ zeolite-based catalysts for the preferential $\mathrm{CO}$ oxidation reaction: Ion-exchange, $\mathrm{Si} / \mathrm{Al}$ and structure effect Catal. Sci. Technol. 62623 
29. Kim S, Lee D W and Lee K Y 2014 Direct synthesis of hydrogen peroxide from hydrogen and oxygen over single-crystal cubic palladium on silica catalysts $\mathrm{J}$. Mol. Catal. A Chem. 383-384 64

30. Mori K, Verma P, Hayashi R, Fuku K and Yamashita H 2015 Color-Controlled Ag Nanoparticles and Nanorods within Confined Mesopores: Microwave-Assisted Rapid Synthesis and Application in Plasmonic Catalysis under Visible-Light Irradiation Chem. - A Eur. J. 2111885

31. Gao D, Zhang X, Dai X, Qin Y, Duan A, Yu Y, Zhuo $\mathrm{H}$, Zhao H, Zhang P, Jiang Y, Li J and Z. Zhao 2016 Morphology-selective synthesis of active and durable gold catalysts with high catalytic performance in the reduction of 4-nitrophenol Nano Res. 93099

32. Sing K S W, Everett D H, Haul R a W, Moscou L, Pierotti R a, Rouquérol J and Siemieniewska T 1982 International union of pure commission on colloid and surface chemistry including catalysis * reporting physisorption data for gas / solid systems with Special Reference to the Determination of Surface Area and Porosity Pure Appl. Chem. 542201

33. Li J, Zhou P, Li F, Ma J, Liu Y, Zhang X, Huo H, Jin J and Ma J 2016 Shape-controlled synthesis of Pd polyhedron supported on polyethyleneimine-reduced graphene oxide for enhancing the efficiency of hydrogen evolution reaction J. Power Sources 302343

34. Jin M, Liu H, Zhang H, Xie Z, Liu J and Xia Y 2011 Synthesis of Pd nanocrystals enclosed by $\{100\}$ facets and with sizes $<10 \mathrm{~nm}$ for application in CO oxidation Nano Res. 483

35. Narayan T C, Baldi A, Koh A L, Sinclair R and Dionne J A 2016 Reconstructing solute-induced phase transformations within individual nanocrystals Nat. Mater. 151
36. Sreedhala S, Sudheeshkumar W and Vinod C P 2016 CO oxidation on large high-index faceted $\mathrm{Pd}$ nanostructures J. Catal. 337138

37. Zheng $\mathrm{W}, \mathrm{Qu} \mathrm{J}$, Hong $\mathrm{X}$, Tedsree $\mathrm{K}$ and Tsang $\mathrm{S}$ C E 2015 Probing the Size and Shape Effects of Cubic- and Spherical-Shaped Palladium Nanoparticles in the Electrooxidation of Formic Acid ChemCatChem 7 3826

38. Niu W, Zhang L and Xu G 2010 Shape-controlled synthesis of single-crystalline palladium nanocrystals ACS Nano 41987

39. Collins G, Schmidt M, McGlacken G P, O'Dwyer C and Holmes J D 2014 Stability, oxidation, and shape evolution of PVP-capped Pd nanocrystals J. Phys. Chem. C 1186522

40. Navlani-García M, Miguel-García I, BerenguerMurcia A, Lozano-Castelló D, Cazorla-Amorós D and Yamashita H $2016 \mathrm{Pd} /$ zeolite-based catalysts for the preferential $\mathrm{CO}$ oxidation reaction: ion-exchange, Si/Al and structure effect Catal. Sci. Technol 6 2623

41. Liu Z, Shamsuzzoha M, Ada E T, Reichert W M and Nikles D E 2007 Synthesis and activation of Pt nanoparticles with controlled size for fuel cell electrocatalysts $J$. Power Sources 164472

42. Rioux R M, Song H, Hoefelmeyer J D, Yang P and Somorjai G A 2005 High-surface-area catalyst design: Synthesis, characterization, and reaction studies of platinum nanoparticles in mesoporous SBA-15 silica J. Phys. Chem. B 1092192

43. Gao D, Zhou H, Wang J, Miao S, Yang F, Wang G, Wang J and Bao X 2015 Size-Dependent Electrocatalytic Reduction of $\mathrm{CO}_{2}$ over Pd Nanoparticles J. Am. Chem. Soc. 137 4288 